\title{
Interactive comment on "Incorporating multi-source remote sensing in the detection of earthquake-damaged buildings based on logistic regression modelling" by Qiang Li et al.
}

Qiang Li et al.

liqiang08@163.com

Received and published: 22 September 2020

Dear reviewer: I am very grateful to your comments for the manuscript. According with your advice, we amended the relevant part in manuscript. Some of your questions were answered below. 1. ABSTRACT. The abstract is very wordy, and lacks, until we get to the last few At no point in the abstract does it talk about which years/how many images, how big an area is studied, but rather is a narrative description of the data. Please make this more of a summary of the manuscript, rather than narrative. Reply: Thank you for your suggestion. We will reorganize and compile the abstract and supplement the relevant main contents. Please refer to the revised manuscript

Printer-friendly version

Discussion paper 
for details 2. English. Throughout, this will need to be checked carefully by the copy editors, but overall, the English is understandable (but English as a second language). Reply: Thank you very much. We will employ a professional language polishing company to modify the language. 3.Paragraph 1 (Introduction). Please add appropriate references (cite other people), rather than just narrative. Reply: Thank you very much. We will supplement the relevant important references in the corresponding position. 4. Introduction (background). I did not feel that you have appropriately reflected the literature of OTHERS that have done work on multi-source remote sensing for earthquake damaged buildings. I would like to suggest either in the introduction, or another section which should be named BACKGROUND (or something similar) you do a much more thorough literature review of those who have worked on examining earthquake damaged buildings based on remote sensing. Ideally, this would be a TABLE with headers that pull out information from these papers, and provides a critical review (it does not have to be at a review paper level, but enough so we have an idea of what has been done before). These headers might be "Source" (e.g., Voigt et al, 2007), Region, Earthquake, Remote Sensing Products Used, : : :, : : :., : : :., Main comments. Then, in the text of the paper, you can refer to this table, and compare and contrast. As it is, the studies you cite tend to be dated $(2007,2009,2011,2011,2006$, 2012, 2011, etc.) with no papers in the introduction which are since 2012. A lot has happened since then, and it does not feel that you are 'building' on others' work by acknowledging them. The overall result is a Master's thesis, and not critically done, in terms of the background. References. Throughout, please go sentence by sentence and ensure that you have referred to the literature. If you have facts, ideas of other people, you need an in-text citation. For example, in Section 2, you do not have any references, but then state items of fact such as the Wenchuan earthquake caused a large number of casualties and damage to facilities (give a reference). Old Beichuan County resulted in relocation of the entire community (needs a reference). There are many similar sentences. You need to be VERY CLEAR where your facts and information that you cite are from. Reply: Thank you very much. We will carefully sort out

Interactive comment
Printer-friendly version

Discussion paper 
the full text and supplement relevant references in the quoted position. Please refer to the revised manuscript for details. 5.Section 2. Study case and Datasets. l'd like a lot more specificity about the study area and the data used. How big is the study area (Old Beichuan County) .what kind of geology is there? Is it an area heavily populated? Density? Lots of buildings? For the datasets, which years/months? How many? You are vague about the data, so person would not be able to repeat what you did (they don't know what you used). Throughout, you need to ensure that the reader knows the (relevant background) to the study area, exactly the data you used, and then what you did with it. Reply: Thank you very much. We will add the following information: The details of the datasets are shown in the following table. The area of Old Beichuan County is $2.66 \mathrm{Km} 2$. MS 8.0 Wenchuan earthquake caused Beichuan County massive destruction,and resulted in casualties more than ten thousand.The whole Beichuan County has become in ruins. Field seismic investigation showed that the three principal causes of such huge destruction are as follows:(1)the vibration failure effect caused by macroseism vibration;(2) the earth's surface rupture effect caused by seismo-active fault slippage; and(3) the secondary geological hazards(collapse,landslide and debris flow) caused by the earthquake. The information of the datasets used in the experiments Datasets Acquisition time Spatial resolution UAV optical multispectral image 2013.10 0.25m Ground-based LiDAR 2013.10 0.5m SAR (TerraSAR) $2014.51 \mathrm{~m}$

6.Section 3. Seismic Characteristics of multi-source remote sensing images. This is fairly description rather than quantitative in its presentation of the seismic characteristics one can detect using remote sensing images. There are some good parts in here, but can the section be made slightly more organized in its structure. This is evident also in having just one reference cited for the entire section. Thas there really been no one else who has looked at seismic characteristics using multi-source remote sensing images? Reply: Thank you. Your suggestion is right. We will adjust the structure of the chapters to make them more logical, and supplement the corresponding references. Sorry about it. We have done the analysis of seismic damage characteristics by ourselves, so we have not quoted other references, but we are sorry that we have not

Printer-friendly version

Discussion paper 
quoted references in the feature calculation. We will supplement and revise them in the revised manuscript. 7.Section 4. Methodology. In terms of structure, this borders on narrative in places and coule be slightly better organized in terms of "We did the following steps: (i) ${ }^{* * *}$, (ii) ${ }^{* * * *}$, (iii) ${ }^{* * * * *}$ with any appropriate references. In terms of content of the methodology, although parts of this are good, imagine someone who does not have your work, trying to now read it and replicate it. Have you put in enough details for that person to reproduce each step. So give this to one of your (student) colleagues NOT familiar with the work, and ask them if they could reproduce each step over an hour. Reply: Sorry about it. In the revised manuscript, we will add the following contents. We did the following steps: (i) Data processing, including optical image, SAR image, DSM image, and building sample distribution , (ii) Feature selection, including the spectral features of optical images, the texture features of SAR images and the geometric features of DSM images (iii) Logistic regression Model construction and analysis. At the same time, in the introduction of each step, the software, process and detailed setting parameters of the method will be supplemented. 8.Section 5 and 6 . l'd like to better understand the behaviour of your results and the uncertainty. So in practice, what would it mean if we were to use your algorithm in another region? Would we get 50Reply: Thanks for your advices. The main purpose of this paper is to construct a method to extract earthquake damage information. Therefore, if this method is used in other areas, only the data meet the conditions, of course, if the data type is not complete, this method can also be used. We will supplement the correct recognition rate and wrong classification rate of earthquake damaged buildings in the revised manuscript, so that we can directly see the accuracy of information identification of this method. Your suggestion is very right. The input content of the method proposed in this paper is mainly image features. When the input features are more comprehensive, the seismic damage information extracted by fitting equation will be more accurate. If the image quality is not very good (for example, there are clouds in the image, the spatial resolution of the image is low), these will also affect the accuracy of information recognition. We will also

Printer-friendly version Discussion paper (n) 
supplement the discussion in the revised version. 9.Equations. Equation 1. Infoterra is an 'interesting' source. Do you genuinely have no other sources of reference for this key equation? What is sigma standing for âË̈̈A ËĞT you have not told us, nor why it is raised to the 0th power (I guess this is your radar sigma naught value). What is $s$ in the

$\mathrm{k}_{s}$.Someproblems forthisandtheotherequationswith formatting.Tellustherangeofvalueshere, andwhyDteresitiviosoluteval Sorryaboutit.Thereferencesisasfollows : Infoterra, RadiometricCalibrationofTerraSAR-

comment

X Data, TS XX-ITD-TN-0049, 2008.ThereferenceisTerraS ARdataproductmanual.Thecalculationmethodisal socited sectionalarea(i.e.backscatteringcoef ficient)perunitarea, whichcanbeusedtocharacterizethescatteringabilityofthetargett Sorryaboutit.Intherevisedmanuscript, wewillstandardizeandunifyabbreviations.11.Variables.Youseemtogobackandfort $600^{\prime \prime}[? m] A^{\prime}$ 'Equationin Section5.2Reply : Sorryaboutit.Wewillcheckandrevisetheunitsofvariables.12.Tgivethisanumber, $B R)+\left(0.419 * C O N^{\prime \prime}\right.$. Reminduswhattheacronymsmean.Reply

Sorryaboutit.Wewilladdrelevantcontents13.Conclusions.I'mnotconvincedwhetherthisisapaperthatreallyisanewmethod. Themethodproposedinthismanuscriptisanewmethodinthe fieldofearthquakedamagein formationidentification.Inthecon ***, by*****).A'cFigure6.VariablesgobackandforthbetweenpandP.Figurecaptionneedstobemorecomplete.Thecolour smad 8.Definewhatyoumeanby $\mathrm{E}, V A, H O M, D I$, etc., inthe figurecaption. Whydif ferentcolours.Poorlydonelabelsinplaces $A$ Sorryaboutit.Wewillunifythestandardforthecaptionandlegendofall figuresinthemanuscript.Especially fortheproblemsr Sorryaboutit.Wewillunifythestandardofalltablesinthemanuscript.

Interactive comment on Nat. Hazards Earth Syst. Sci. Discuss., https://doi.org/10.5194/nhess2019-20, 2019. 